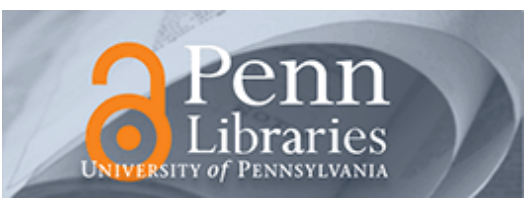

University of Pennsylvania

ScholarlyCommons

\title{
Single-Negative, Double-Negative, and Low-index Metamaterials and their Electromagnetic Applications
}

\author{
Andrea Alù \\ University of Pennsylvania \\ Nader Engheta \\ University of Pennsylvania, engheta@ee.upenn.edu
}

\author{
A. Erentok \\ University of Arizona
}

Richard W. Ziolkowski

University of Arizona

Follow this and additional works at: https://repository.upenn.edu/ese_papers

Part of the Electromagnetics and Photonics Commons

\section{Recommended Citation}

Andrea Alù, Nader Engheta, A. Erentok, and Richard W. Ziolkowski, "Single-Negative, Double-Negative, and Low-index Metamaterials and their Electromagnetic Applications", . February 2007.

Copyright 2007 IEEE. Reprinted from IEEE Transactions on Antennas and Propagation Magazine, Volume 49, Issue 1, February 2007, pages 23-36.

This material is posted here with permission of the IEEE. Such permission of the IEEE does not in any way imply IEEE endorsement of any of the University of Pennsylvania's products or services. Internal or personal use of this material is permitted. However, permission to reprint/republish this material for advertising or promotional purposes or for creating new collective works for resale or redistribution must be obtained from the IEEE by writing to pubs-permissions@ieee.org. By choosing to view this document, you agree to all provisions of the copyright laws protecting it.

This paper is posted at ScholarlyCommons. https://repository.upenn.edu/ese_papers/258

For more information, please contact repository@pobox.upenn.edu. 


\title{
Single-Negative, Double-Negative, and Low-index Metamaterials and their Electromagnetic Applications
}

\author{
Abstract \\ Metamaterials that are engineered media characterized by electromagnetic constitutive parameters with \\ anomalous values may show counterintuitive properties in their interactions with electromagnetic waves. \\ Here, we review some of the properties and potential applications we have recently presented in the \\ technical literature: properties and applications in which plasmonic materials and metamaterials may be \\ utilized to overcome some conventional physical limits. Resonances arising in electrically small regions \\ of interface where these materials are paired with common materials are shown to be potentially \\ attractive for this purpose in some electromagnetic problems, for instance, in guiding and radiating \\ structures. The anomalous refractive properties at such "complementary" interfaces and the negative \\ values of polarizability attainable in such materials are also shown to offer potentials for several \\ applications.

\section{Keywords} \\ metamaterials, permittivity, permeability, composite materials, resonators, waveguides, antennas, \\ negative index material, negative refraction

\section{Disciplines} \\ Electromagnetics and Photonics

\section{Comments} \\ Copyright 2007 IEEE. Reprinted from IEEE Transactions on Antennas and Propagation Magazine, Volume \\ 49, Issue 1, February 2007, pages 23-36. \\ This material is posted here with permission of the IEEE. Such permission of the IEEE does not in any way \\ imply IEEE endorsement of any of the University of Pennsylvania's products or services. Internal or \\ personal use of this material is permitted. However, permission to reprint/republish this material for \\ advertising or promotional purposes or for creating new collective works for resale or redistribution must \\ be obtained from the IEEE by writing to pubs-permissions@ieee.org. By choosing to view this document, \\ you agree to all provisions of the copyright laws protecting it.
}




\title{
Single-Negative, Double-Negative, and Low-Index Metamaterials and their Electromagnetic Applications
}

\author{
A. Alù ${ }^{1}$, N. Engheta ${ }^{1}$, A. Erentok ${ }^{2}$, and R. W. Ziolkowski ${ }^{2}$ \\ ${ }^{1}$ University of Pennsylvania, Department of Electrical and Systems Engineering \\ 200 South 33rd St., Philadelphia, PA 19104, USA \\ Tel: +1 (215) 898-9777; Fax: +1 (215) 573-2068; E-mail: engheta@ee.upenn.edu \\ ${ }^{2}$ University of Arizona, Department of Electrical and Computer Engineering \\ 1230 E. Speedway Blvd., Tucson, AZ 85721, USA \\ Tel: +1 (520) 621-6173; Fax: +1 (520) 621-8076; E-mail: ziolkowski@ece.arizona.edu
}

\begin{abstract}
Metamaterials that are engineered media characterized by electromagnetic constitutive parameters with anomalous values may show counterintuitive properties in their interactions with electromagnetic waves. Here, we review some of the properties and potential applications we have recently presented in the technical literature: properties and applications in which plasmonic materials and metamaterials may be utilized to overcome some conventional physical limits. Resonances arising in electrically small regions of interface where these materials are paired with common materials are shown to be potentially attractive for this purpose in some electromagnetic problems, for instance, in guiding and radiating structures. The anomalous refractive properties at such "complementary" interfaces and the negative values of polarizability attainable in such materials are also shown to offer potentials for several applications.
\end{abstract}

Keywords: Metamaterials; permittivity; permeability; composite materials; resonators; waveguides; antennas; negative index material; negative refraction

\section{Introduction}

$\mathbf{N}$ atural plasmonic materials (noble metals and some dielectrics), the electric resonances in the microscopic molecular domain of which induce an overall negative electric permittivity for the bulk medium at optical frequencies, are known to show an interesting and anomalous electromagnetic response in the visible regime [1]. In a similar way, by mimicking the molecular functions that cause these anomalous resonances, but scaled at lower frequencies, metamaterials with nonstandard values of their constitutive parameters have recently been conceptually proposed and synthesized by properly embedding suitably shaped inclusions in a given host material [2]. Nowadays, advances in simulation and fabrication technologies allow a rather broad flexibility in the design of these metamaterials and, hence, their electromagnetic responses. The potential ability to engineer the electromagnetic responses of materials for a wide variety of applications has stimulated significant interest in metamaterials. Interestingly

"This is an updated version of A. Alù, N. Engheta, A. Erentok, and R. W. Ziolkowski, "Single-Negative, Double-Negative, and LowIndex Metamaterials and their Electromagnetic Applications," Radio Science Bulletin, No. 319, December, 2006, pp. 6-19. enough, the recent advances in nanotechnology and molecular bioengineering are leading researchers to speculate about the possibility of bringing these metamaterial concepts back to the visible frequencies, and about the proper design of artificial molecular shapes to achieve artificial optical metamaterials, in order to tailor their electromagnetic properties at infrared and visible frequencies.

If we assume that the material response is isotropic - at least for a given polarization of the fields and in a specific range of frequencies - and that the magneto-electric coupling is negligible, the time-harmonic Maxwell's equations (with an $e^{j \omega t}$ time dependence), in the absence of impressed sources, are locally written as

$$
\begin{aligned}
& \nabla \times \mathbf{E}=-j \omega \mu \mathbf{H}, \\
& \nabla \times \mathbf{H}=j \omega \varepsilon \mathbf{E},
\end{aligned}
$$

where $\varepsilon, \mu$ respectively represent the local electric permittivity and magnetic permeability, and are complex quantities when losses are taken into account. From their electromagnetic wave interactions, most of the materials in nature are characterized by these two quantities yielding values compatible with the constraints $\operatorname{Re}[\varepsilon] \geq \varepsilon_{0}, \operatorname{Re}[\mu] \geq \mu_{0}, \operatorname{Im}[\varepsilon]<0, \operatorname{Im}[\mu]<0$, which imply the 
passive nature of the material and an index of refraction greater than or equal to the free-space value. In principle, however, at a given frequency, the real parts of the permittivity and permeability of a passive material may yield any real value, provided a suitable temporal dispersion satisfies the constraints dictated by causality (i.e., the Kramers-Krönig relations [3]). Materials with the simple constitutive relations described by Equation (1) may be categorized according to the diagram of Figure 1: if both the permittivity and permeability have positive real parts, as most of the materials in nature do, they may be called "double-positive (DPS)" media, whereas if both of these quantities are negative, as in the third quadrant of Figure 1, the corresponding materials may be called "double-negative (DNG)" [4]. Owing to their anomalous wave refraction, such materials have been the subject of great interest in the engineering and physics communities, particularly since their experimental realization by the UCSD group in 2000 [2]. Media with a negative real part of the permittivity, but a positive permeability, are located in the second quadrant, and are named " $\varepsilon$ negative (ENG)." They include plasma and plasmonic materials (noble metals and some polar dielectrics) below their plasma frequencies. Applications of plasmas and other forms of $\varepsilon$-negative materials in several different fields have been studied for decades. Recently, due to the development in nanotechnologies, there has been a renewed interest in the plasmonic resonances associated with sub-wavelength particles and interfaces. In the fourth quadrant, we have the $\mu$-negative (MNG) materials, which may be realized with ferromagnetic materials or synthesized with suitable inclusions in a host material [5]. The artificially-realized $\mu$-negative materials are essential, basic constituents in the construction of double-negative materials. In analogy with double-negative materials, $\varepsilon$-negative and $\mu$-negative materials may be collectively named "single-negative (SNG)" media. Near the two axes of Figure 1 , where the real part of one of the constitutive parameters is near zero, the materials may be termed $\varepsilon$-near-zero (ENZ) and $\mu$ near-zero (MNZ) materials, depending on the constitutive parameter that yields anomalous values. Materials with both constitutive parameters equal to zero, which fall at the origin of Figure 1 , have been termed zero-index materials [6]. We note that the realization of single-negative materials may be relatively easier than that of double-negative materials, and therefore particular efforts are recently being aimed at exploring how some of the exciting phenomena and applications predicted and studied in double-negative materials can be transferred into single-negative media.

As underlined above, low or negative values of the effective constitutive parameters may be properly tailored at specific frequencies, but they are associated with dispersive media [7]. In passive materials, the frequency dispersion must also obey some specific constraints that are related to the causality and energy relations. In particular, in the low-loss scenario, even though $\varepsilon, \mu$ may be both negative quantities, their frequency behavior should satisfy the conditions:

$$
\begin{aligned}
& \frac{\partial(\omega \varepsilon)}{\partial \omega} \geq 0, \\
& \frac{\partial(\omega \mu)}{\partial \omega} \geq 0 .
\end{aligned}
$$

This implies that the total electromagnetic energy in the material must be positive. In the following, we review some of the potential applications of metamaterials, recently proposed and reported in the literature. Our analysis of these different problems takes into account the possible dispersion and loss characteristics of the metamaterials; the associated detrimental effects are reduced by properly choosing the operating frequencies in regions in which their influence may be less drastic.

\section{Applications of Negative Materials}

\subsection{Sub-Wavelength Cavities, Resonators, and Waveguides}

The double-positive and double-negative materials support monochromatic plane wave propagation in the form $e^{j(\omega t-k z)}$, since their wave vector, $k=\omega \sqrt{\varepsilon} \sqrt{\mu}$, is a real number in the limit of no losses. In particular, the wave vector and Poynting vectors are parallel in a double-positive material, whereas in a doublenegative material they are oppositely directed, implying backwardwave phase propagation and negative phase velocity for a wave carrying power in the positive direction. The $\varepsilon$-negative and $\mu$ negative materials, on the other hand, are opaque to radiation, since they support only evanescent waves, i.e., $k$ is imaginary. As was stated in [8], when employed by themselves, even the most awkward negative materials do not show particularly appealing properties: "The unconventional electromagnetic characteristics of metamaterials are exhibited when these materials are paired with other materials with at least one oppositely-signed constitutive parameter" [8]. In fact, the anomalies in employing materials with negative constitutive parameters arise at the interface between two media with at least one pair of oppositely signed parameters. This can play a major role in the anomalous behavior of the combined structure. In fact, at the boundary between such media, the continuity of the tangential electric and magnetic field components implies

$$
\begin{aligned}
& \left.\frac{1}{-j \omega \mu_{1}} \frac{\partial E_{1, \tan }}{\partial n}\right|_{\text {Interface }}=\left.\frac{1}{-j \omega \mu_{2}} \frac{\partial E_{2, \tan }}{\partial n}\right|_{\text {Interface }}, \\
& \left.\frac{1}{j \omega \varepsilon_{1}} \frac{\partial H_{1, \tan }}{\partial n}\right|_{\text {Interface }}=\left.\frac{1}{j \omega \varepsilon_{2}} \frac{\partial H_{2, \tan }}{\partial n}\right|_{\text {Interface }},
\end{aligned}
$$

where $\partial / \partial n$ is the normal derivative with respect to the interface, and $\varepsilon$ and $\mu$ are the permittivity and permeability in the two media. It is clear that when $\mu_{1}$ and $\mu_{2}$, and/or $\varepsilon_{1}$ and $\varepsilon_{2}$ have opposite signs, i.e., at the interface between $\varepsilon$-negative, $\mu$-negative double-negative and/or double-positive materials, the derivatives of the tangential fields on both sides of this interface will have opposite signs. This peculiar "V-shaped" discontinuity in the derivative is a symptom of a concentrated resonant phenomenon at this "complementary" interface (similar to the current and voltage distributions at the junction between an inductor and a capacitor at the resonance of an $L-C$ circuit).

The possibility of designing thin, sub-wavelength cavity resonators and parallel-plate waveguides, in which a layer of double-negative, $\varepsilon$-negative or $\mu$-negative material is paired with a double-positive layer, or in which $\varepsilon$-negative and $\mu$-negative materials are paired together, has been suggested following these premises $[9-12,13]$. This compact resonance, together with the phase-compensation typical of negative materials, in fact allows resonant modes in electrically thin parallel-plate structures containing such bi-layered fillings to be obtained. An example is pro- 


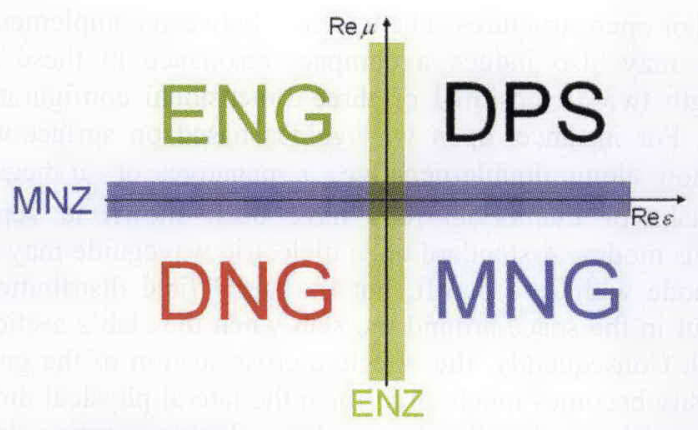

Figure 1. The nomenclature of metamaterials, based on the values of the real parts of their permittivity and permeability.

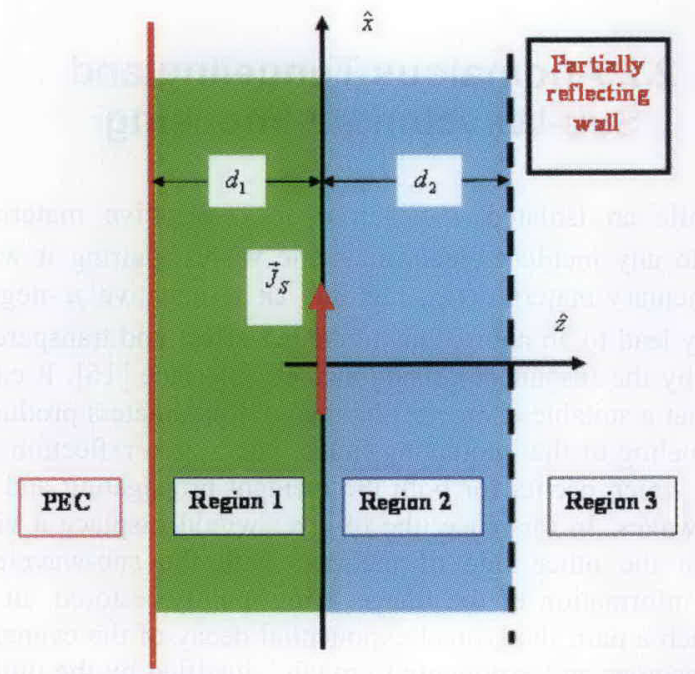

Figure 3a. The geometry of an ultra-thin laser cavity for a double-positive/double-negative and an $\varepsilon$-negative/- $\mu$-negative bi-layer. The source is sandwiched between two metamaterial slabs that are terminated with a PEC and a partially reflecting mirror (from [12], C2006 Optical Society of America).

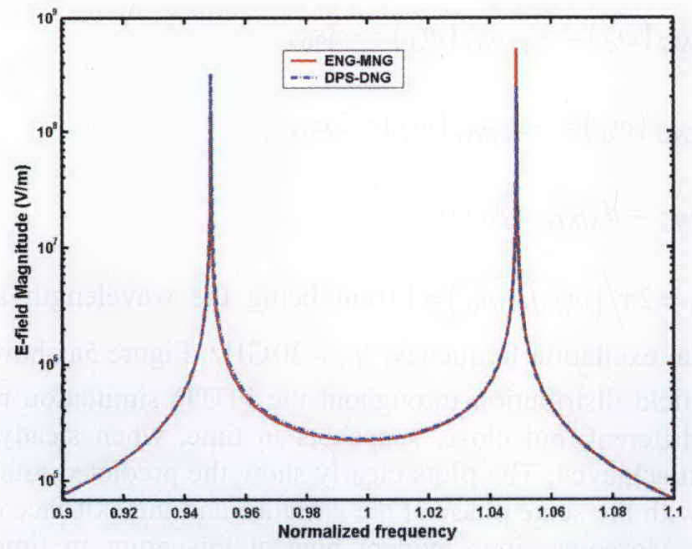

Figure 3b. The output electric-field magnitudes for the geometry of Figure 3a (from [12], (C2006 Optical Society of America).

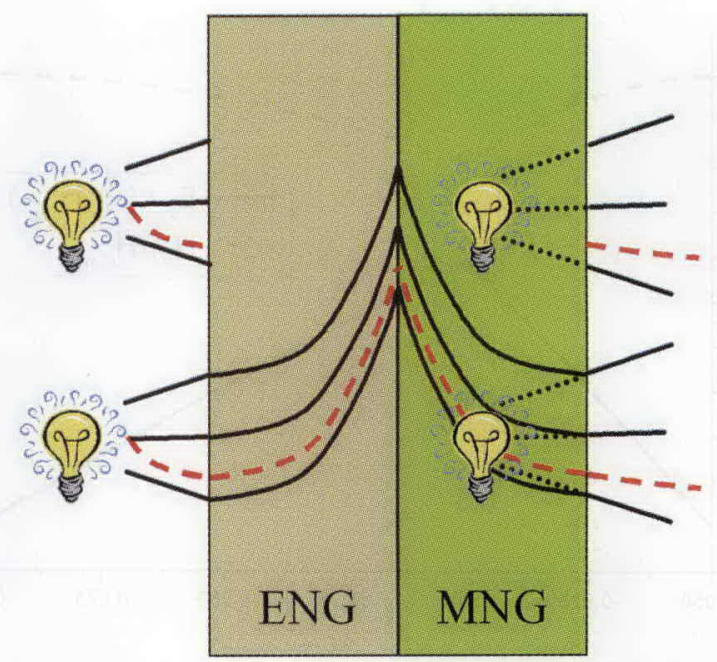

Figure 4. A schematic model of the virtual displacer made with a complementary $\varepsilon$-negative/ $\mu$-negative bi-layer. Propagating and evanescent waves that construct a given object on the entrance face of the bi-layer (represented, respectively, by the black rays and the red exponential curve) are restored as a virtual image on its exit face, including all of their sub-wavelength details.

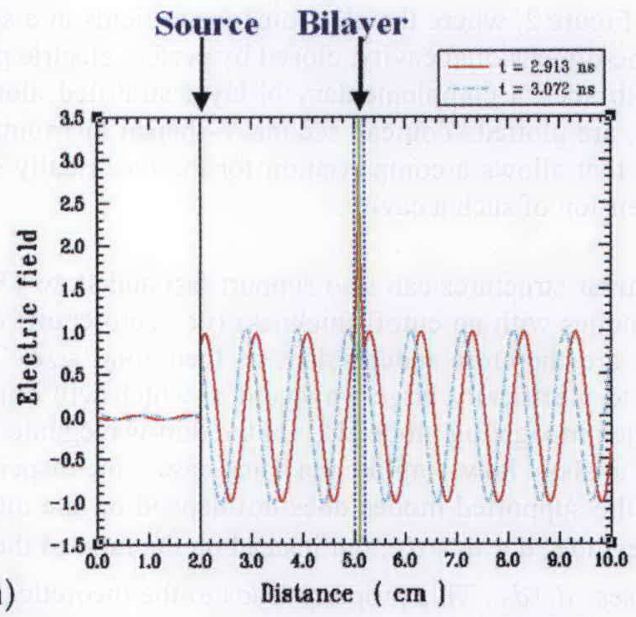

Figure 5a. The predicted anomalous tunneling through a complementarily-matched $\varepsilon$-negative- $\mu$-negative bi-layer was confirmed with one-dimensional FDTD simulations: the electric-field distribution at two snapshots in time.

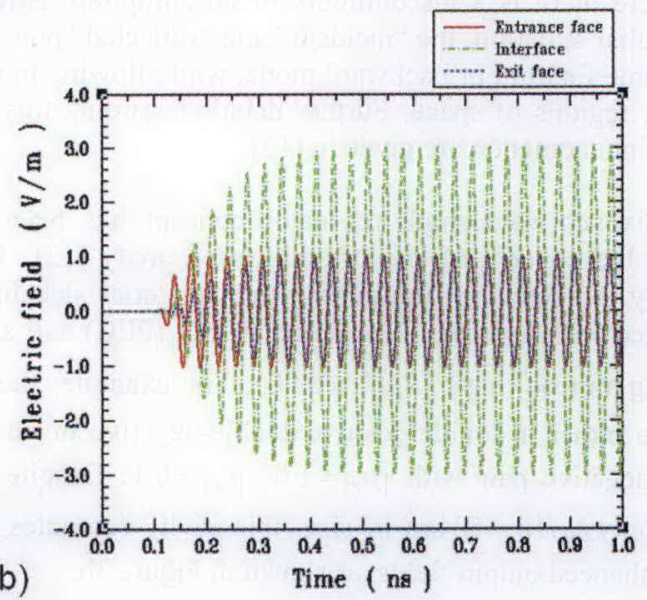

Figure $5 \mathrm{~b}$. The electric-field time histories for the bi-layer of Figure 5a. 


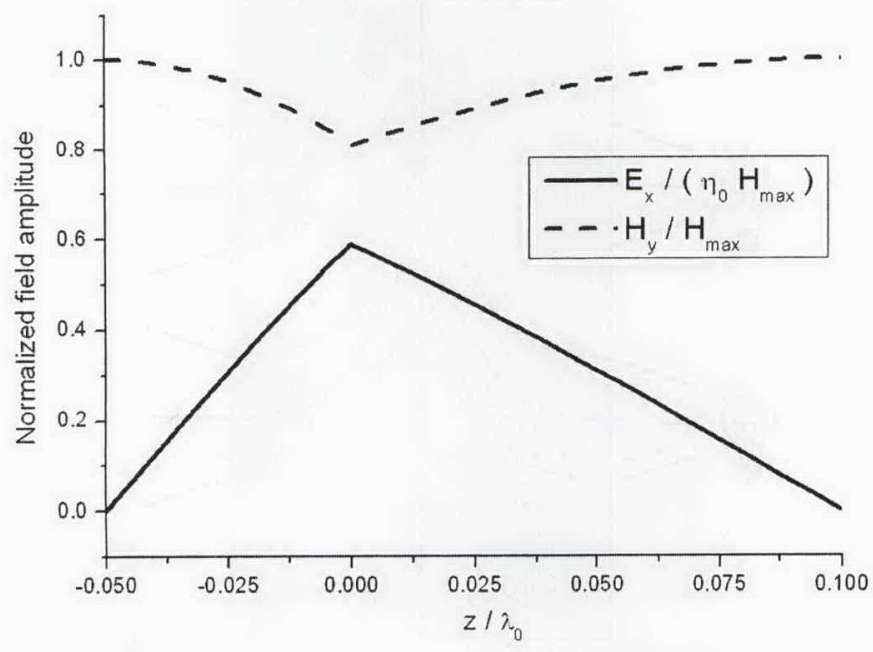

Figure 2. The electric and magnetic field distributions for a one-dimensional sub-wavelength cavity, closed by perfectly conducting walls and partially filled with double-negative metamaterials (the stratification is along the $z$ axis) (from [11]).

vided in Figure 2, where the electromagnetic fields in a sub-wavelength one-dimensional cavity, closed by perfect electric plates and filled with such a complementary bi-layer stratified along the $z$ direction, are plotted. You can see the V-shaped distribution at the interface that allows a compensation for the electrically small lateral dimension of such a cavity.

Similar structures can also support fast and slow TE and TM guided modes with no cutoff thickness (i.e., zero cutoff thickness) and that are therefore independent of their total size. This is in contrast to electrically large waveguides, which will only support one single propagating mode [10]. In the thin-waveguide limit, it is possible to show how - unlike the usual case - the dispersion relation for the supported modes does not depend on the total size of the waveguide, $d=d_{1}+d_{2}$, but instead on the ratio of the two slab thicknesses, $d_{1} / d_{2}$. This property leads to the theoretical possibility of having waveguides supporting a resonant mode even when the total thickness becomes much less than the wavelength. The peculiar flow of energy in such waveguides consists of two antiparallel flows, one each in the two material slabs. This "incident" and "reflected" behavior happens in any standing-wave phenomenon where there is a discontinuity or an abruption. However, in this peculiar situation, the "incident" and "reflected" power belong to the same forward or backward mode, while flowing in distinctly different regions of space. Further details regarding this counterintuitive phenomenon are given in [13].

This sub-wavelength resonator concept has been used to advance the concept of an ultra-thin laser cavity [12]. The basic geometry is shown in Figure 3a: a metamaterial slab bi-layer is terminated with a perfect electric conductor (PEC) and a partially reflecting mirror (e.g., $\left|R_{M}\right|^{2}=0.98$ ). For example, the bi-layer could be either a double-positive/double-negative or an $\varepsilon$-negative/ $\mu$-negative pair with $\left|\mu_{r}\right|=1.0,\left|\varepsilon_{r}\right|=0.1$. Despite a length that is only $\lambda_{0} / 10=50 \mathrm{~nm}$ in size, this cavity resonates and produces enhanced output fields, as shown in Figure $3 \mathrm{~b}$.

Similar sub-wavelength resonant cavities and waveguides may also be envisioned in cylindrical or spherical geometries, both as closed or open structures. The interface between complementary materials may also induce a compact resonance in these subwavelength two-dimensional or three-dimensional configurations $[14,15]$. For instance, open waveguides based on surface-wave propagation along double-negative, $\varepsilon$-negative, or $\mu$-negative planar slabs or cylindrical rods have been shown to support anomalous modes. A standard open dielectric waveguide may support a mode with zero cutoff, but its lateral field distribution is spread out in the space around the slab when the slab's section is too small. Consequently, the effective cross section of the guided mode easily becomes much larger than the lateral physical dimension of the slab. On the other hand, when a double-negative slab or rod is considered, highly concentrated guided modes that propagate in the material are still possible even for very thin doublenegative slabs. This leads to the possibility of building ultra-thin open waveguides, overcoming the standard diffraction limitation in energy transport and reducing the crosstalk between densely packed waveguides [15].

\subsection{Anomalous Tunneling and Sub-Wavelength Focusing}

While an isolated $\varepsilon$-negative or $\mu$-negative material is opaque to any incident electromagnetic waves, pairing it with a complementary material (i.e., building an $\varepsilon$-negative/ $\mu$-negative pair) may lead to an anomalous tunneling effect and transparency, induced by the resonance arising at their interface [16]. It can be shown that a suitable choice of the material parameters produces a total tunneling of the interacting fields, i.e., a zero reflection from the pair, which occurs for both the incident propagating and evanescent waves. In this case, the bi-layer would displace a virtual image on the other side of the pair with the sub-wavelength detailed information of the image being mainly restored. In fact, inside such a pair, the typical exponential decay of the evanescent waves becomes an "exponential growth," justified by the multiple reflections that result from the resonant interface. This anomalous behavior clearly overcomes the intrinsic resolution limitations of any standard image displacer [16]. In Figure 4, a schematic of the functionality of this flat bi-layer as an image displacer is depicted.

The anomalous tunneling behavior of these bi-layers has been studied in both the frequency and time domains [17]. For example, a Finite-Difference Time-Domain (FDTD) engine was used to simulate the initial transient response of the system and its convergence towards the steady-state conditions. The conjugatematched pair had the material parameters

$$
\begin{aligned}
& \varepsilon_{E N G}\left(\omega_{d}\right)=-\varepsilon_{M N G}\left(\omega_{d}\right)=-3 \varepsilon_{0}, \\
& \mu_{E N G}\left(\omega_{d}\right)=-\mu_{M N G}\left(\omega_{d}\right)=2 \mu_{0}, \\
& d_{E N G}=d_{M N G}=\lambda_{0} / 10,
\end{aligned}
$$

with $\lambda_{0}=2 \pi /\left(\omega_{d} \sqrt{\varepsilon_{0} \mu_{0}}\right)=1.0 \mathrm{~cm}$ being the wavelength at the sinusoidal excitation frequency, $f_{0}=30 \mathrm{GHz}$. Figure 5 a shows the electric-field distribution throughout the FDTD simulation region at two different, but close, snapshots in time, when steady-state had been achieved. The plots clearly show the predicted total tunneling, with the same phase at the entrance and the exit face of the bi-layer. Moreover, it is evident how at this point in time, the "growing-exponential" distribution was already present at the $\varepsilon$ negative/ $\mu$-negative interface. This was consistent with the fact 


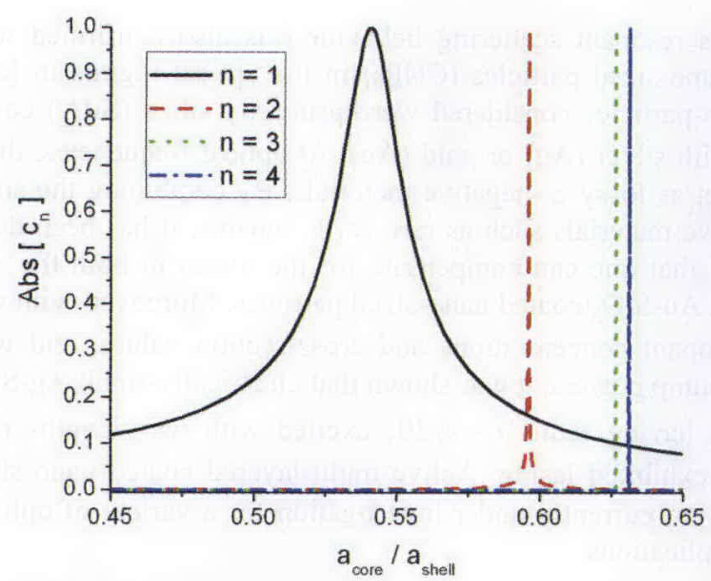

Figure 6. The absolute values of the scattering coefficient, $c_{n}^{T M}$, as defined in [18], for a spherical core-shell system with outer radius $a_{\text {shell }}=\lambda_{0} / 20$, core permittivity $\varepsilon_{\text {core }}=10 \varepsilon_{0}$, and shell permittivity $\varepsilon_{\text {shell }}=-1.2 \varepsilon_{0}$, as a function of the filling ratio of the sphere (reprinted with permission from [18]; copyright 2005 American Institute of Physics).

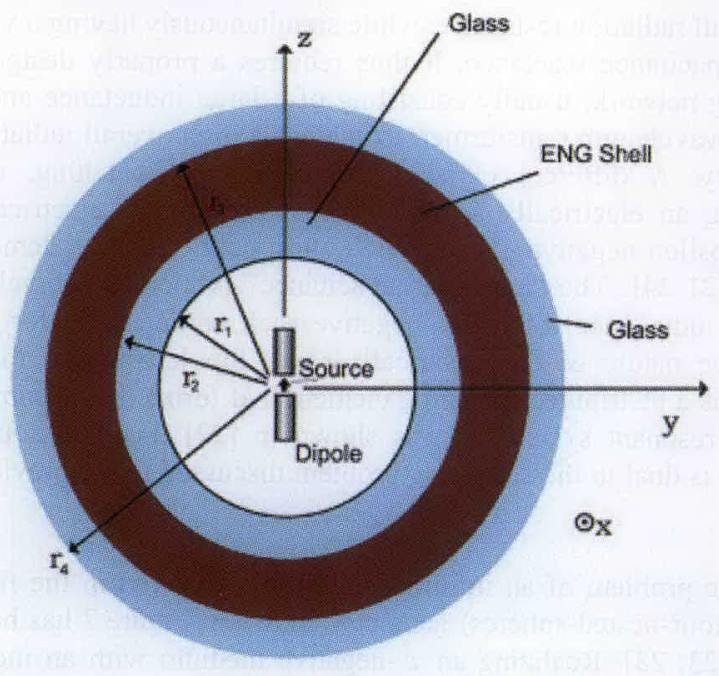

Figure 7. An electric dipole antenna surrounded by a concentric multilayered spherical shell that consists of two glass shells surrounding an $\varepsilon$-negative shell.

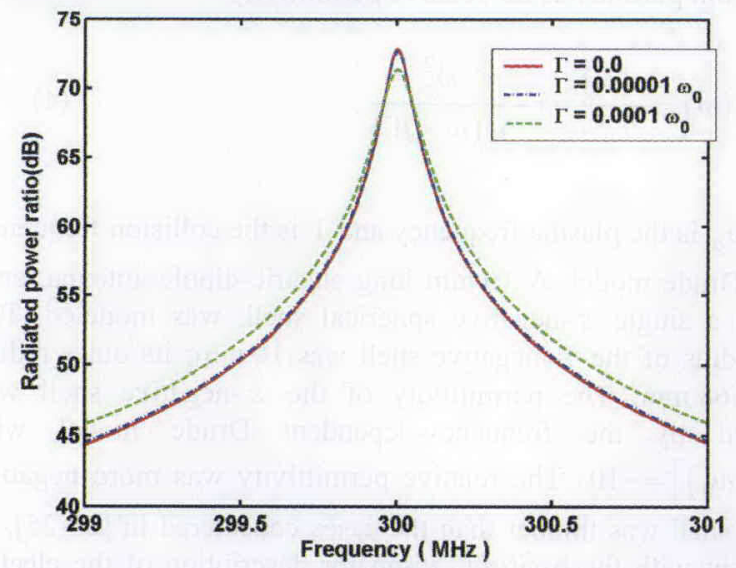

Figure 8a. Enclosing an infinitesimal dipole antenna in a resonant electrically-small single-layer $\varepsilon$-negative shell significantly enhances the power radiated by the antenna for a given constant driving current. A plot of the radiated power ratio, assuming that the permittivity of the shell is described by a Drude model.

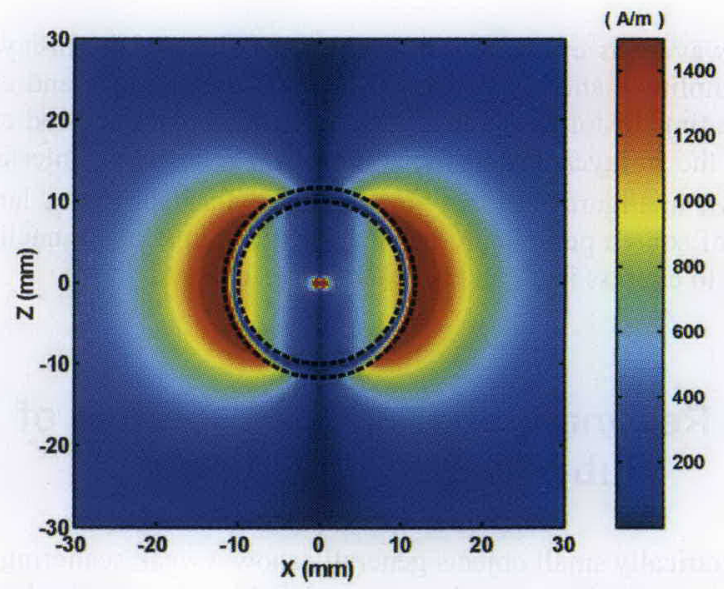

Figure 8b. The magnetic-field distribution for the lossless case of the infinitesimal dipole antenna in a resonant electricallysmall single-layer $\varepsilon$-negative shell as in Figure 8a.

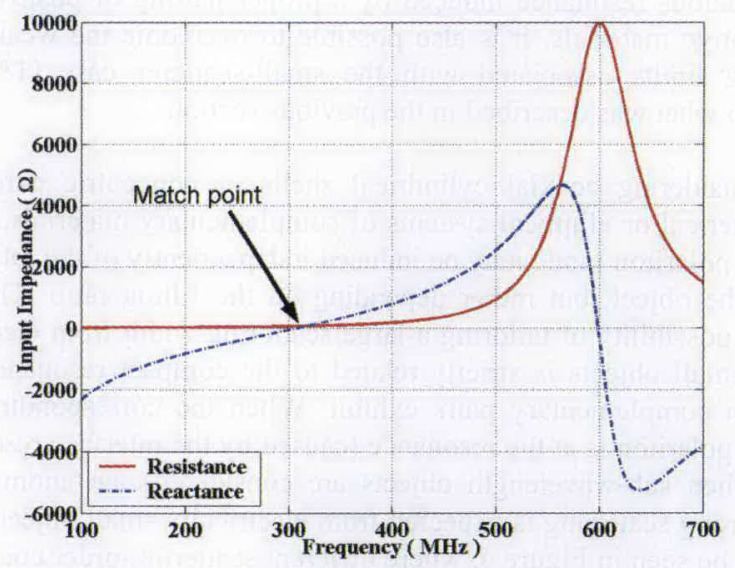

Figure 9. The input impedance predicted by HFSS for a center-fed dipole enclosed in a glass-plasma-glass system, fed with a $1 \mathrm{~W}, 75 \Omega$ source, assuming that the shell's permittivity is frequency independent and all of the layers are lossless.

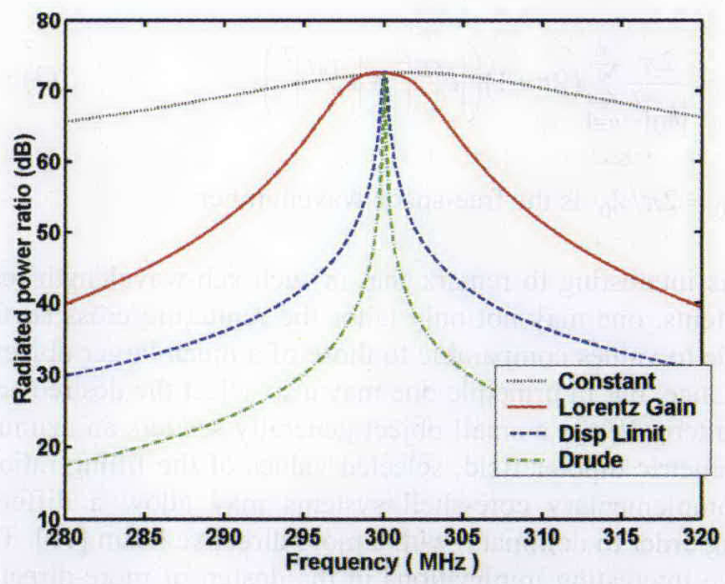

Figure 10. The radiated power ratio (RPR) of an electrically small dipole antenna driven with a fixed current and surrounded by an electrically small matched $\varepsilon$-negative spherical shell, the permittivity of which is described by several designed dispersion models. 
that the wave was evanescent in each of the slabs of the bi-layer, but its amplitude and phase was the same at the entrance and exit face. The time histories of the electric field at the entrance and exit faces of the bi-layer and at the $\varepsilon$-negative/ $\mu$-negative interface are shown in Figure $5 \mathrm{~b}$. They demonstrated the fact that a large number of source periods are needed for the anomalous tunneling behavior to express itself and to reach its steady state.

\subsection{Resonant Scattering Properties of Sub-Wavelength Objects}

Electrically small objects generally show a weak scattering of any incident radiation, and the scattered fields are strongly dominated by the dipolar component. A peak in the scattering cross section of an object is in fact associated with a polariton resonance of its structure, similar to an open cavity at its resonance frequency. Usually such resonant modes can be excited in large objects, with sizes that are at least comparable with the wavelength, as occurs in any standard cavity. However, by exploiting the anomalous resonance induced by a proper pairing of positive and negative materials, it is also possible to overcome the weakscattering limits associated with the small-scatterer case [18], similar to what was described in the previous section.

Considering coaxial cylindrical shells or concentric coreshell spherical or elliptical systems of complementary materials, a resonant polariton mode may be induced independently of the total size of the object, but rather depending on the filling ratio. The intrinsic possibility of tailoring a large scattering width from electrically small objects is strictly related to the compact resonance that such complementary pairs exhibit. When the corresponding material polariton is at the resonance (caused by the interface resonance when sub-wavelength objects are considered), an anomalously strong scattering is expected from electrically small objects. This can be seen in Figure 6, where different scattering-order coefficients $c_{n}^{T M}$ (as classically defined, see [18]) are plotted for an example of a double-positive/ $\varepsilon$-negative core-shell sub-wavelength sphere as functions of the filling ratio of the two materials. We note how for specific ratios, one of the scattering orders reaches its absolute maximum (which is when $c_{n}^{T M}=-1$, at a polariton resonance), leading to a resonant total scattering cross section, which is given by the standard expression

$$
Q_{s}=\frac{2 \pi}{\left|k_{0}\right|^{2}} \sum_{n=1}^{\infty}(2 n+1)\left(\left|c_{n}^{T E}\right|^{2}+\left|c_{n}^{T M}\right|^{2}\right)
$$

where $k_{0}=2 \pi / \lambda_{0}$ is the free-space wavenumber.

It is interesting to remark that in such sub-wavelength resonant systems, one may not only tailor the scattering cross section amplitude to values comparable to those of a much larger object at its resonance, but in principle one may also select the desired scattering pattern. While a small object generally scatters an azimuthally symmetric dipolar field, selected values of the filling ratio in these complementary core-shell systems may allow a different scattering order to dominate, with a more directive beam [18]. This may have interesting implications in the design of more-directive radiators with a small electric aperture, due to the excitation of higher-order resonant modes, as suggested in [19]. In principle, any order may be excited at resonance, even though the presence of ohmic losses and the narrow frequency bandwidth may eventually limit this possibility.
This resonant scattering behavior was also confirmed with coated nano-sized particles (CNPs) in the optical regime in [20]. The nano-particles considered were primarily silica $\left(\mathrm{SiO}_{2}\right)$ cores, coated with silver $(\mathrm{Ag})$ or gold $(\mathrm{Au})$. At optical frequencies, these metals act as lossy $\varepsilon$-negative materials. By combining the silica with active materials such as rare-earth dopants, it has been demonstrated that one can compensate for the losses in both the Ag$\mathrm{SiO}_{2}$ and $\mathrm{Au}-\mathrm{SiO}_{2}$ coated nano-sized particles. Moreover, with sufficient dopant concentrations and cross-section values, and with modest pump powers, it was shown that electrically-small $\mathrm{Ag}-\mathrm{SiO}_{2}$ particles, having radii $a \sim \lambda / 20$, excited with wavelengths near $510 \mathrm{~nm}$, exhibited lasing. Active multi-layered coated nano-sized particles are currently under investigation for a variety of opticalbased applications.

\subsection{Resonant Enhancement of the Power Radiated by Electrically Small Antennas}

An electrically small dipole antenna is known to have a large impedance mismatch to any realistic power source, i.e., it has a very small radiation resistance, while simultaneously having a very large capacitance reactance. It thus requires a properly designed matching network, usually consisting of a large inductance and a quarter-wavelength transformer, to achieve a high overall radiation efficiency. A different paradigm for achieving matching, i.e., enclosing an electrically small dipole antenna in an electrically small epsilon-negative (ENG) shell, has been recently demonstrated [21-24]. The "negative capacitance" (which is equivalent with an inductance) of the $\varepsilon$-negative shell compensates for the capacitive nature of the electrically small dipole antenna, functioning as a distributed matching element that forms this "geometrically" resonant system. It was shown in [22] that this source problem is dual to the scattering problem discussed in the previous section.

The problem of an infinitesimal dipole centered in the fiveregion (four-nested-spheres) geometry shown in Figure 7 has been solved $[23,23]$. Realizing an $\varepsilon$-negative medium with an inclusion-based metamaterial, without using an array of infinite wires at UHF frequencies, is very challenging. However, it is straightforward (in principle) with a plasma. An $\varepsilon$-negative shell modeled as a lossy cold plasma has the relative permittivity

$$
\varepsilon_{r}(\omega)=\frac{\varepsilon(\omega)}{\varepsilon_{0}}=1-\frac{\omega_{p}^{2}}{\omega(\omega-j \Gamma)},
$$

where $\omega_{p}$ is the plasma frequency and $\Gamma$ is the collision frequency of this Drude model. A $10 \mathrm{~mm}$ long electric-dipole antenna, centered in a single $\varepsilon$-negative spherical shell, was modeled. The inner radius of the $\varepsilon$-negative shell was $10 \mathrm{~mm}$; its outer radius was $11.68 \mathrm{~mm}$. The permittivity of the $\varepsilon$-negative shell was described by the frequency-dependent Drude model with $\operatorname{Re}\left[\varepsilon_{r}\left(\omega_{0}\right)\right]=-10$. The relative permittivity was more negative and the shell was thinner than the cases considered in [23-25], in agreement with the basic $L C$ resonator description of the electrically small dipole (capacitive element) $\varepsilon$-negative shell (inductive element) system given in [23]. The radiated power ratio (RPR) (the ratio of the total power radiated by the dipole in the presence of the metamaterial shells and in free space for the same constant driving current, e.g., $1 \mathrm{~A}$ ) was obtained for various values of the collision frequency: these results are shown in Figure 8a. The magnitude of 


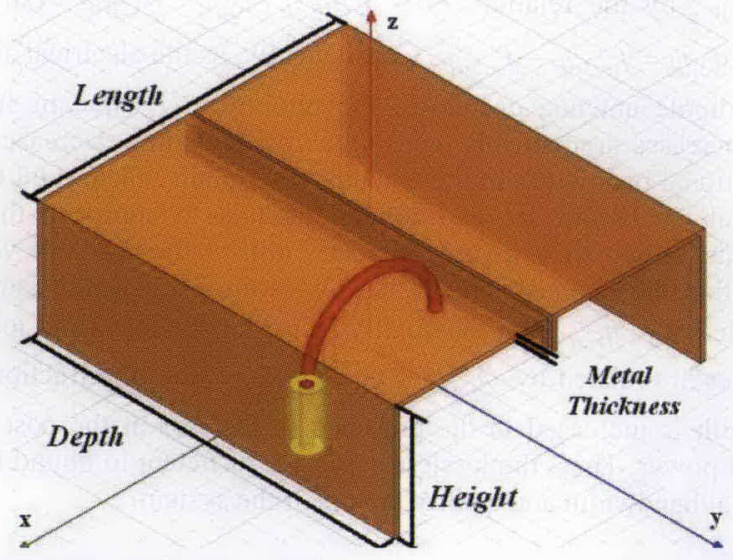

Figure 11a. The three-dimensional EZ-magnetic antenna geometry.

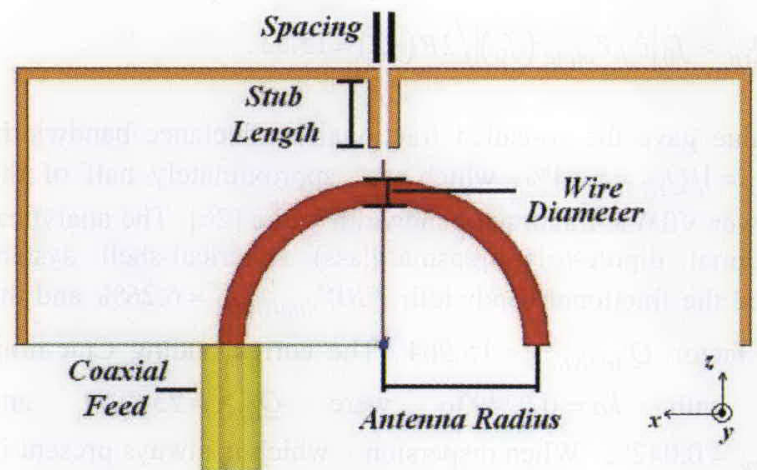

Figure 11b. The detailed specifications of each design variable for the three-dimensional EZ-magnetic antenna.

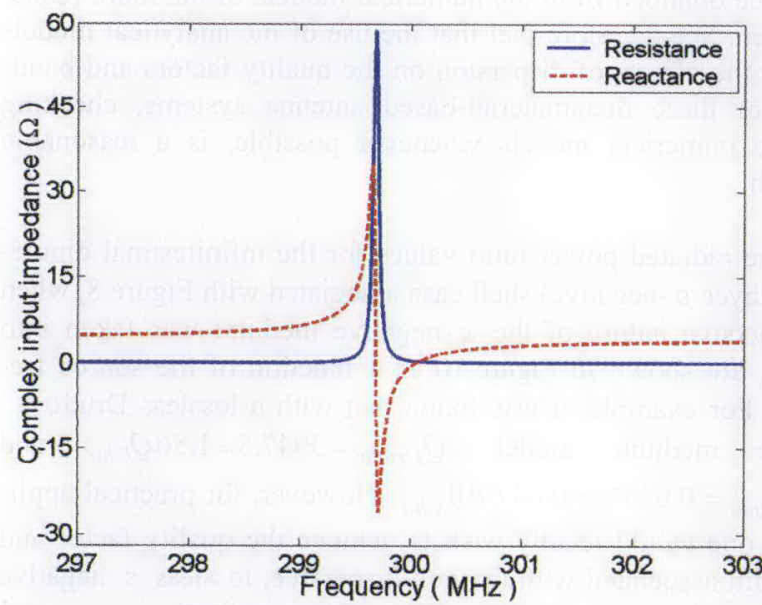

Figure 12a. The complex input impedance values for Design 1.

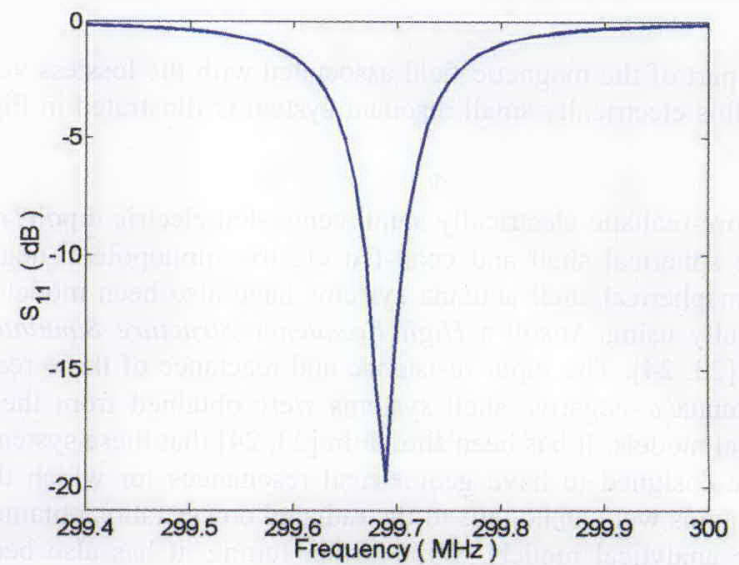

Figure 12b. The predicted $S_{11}$ values calculated for a 50-ohm source for Design 1.

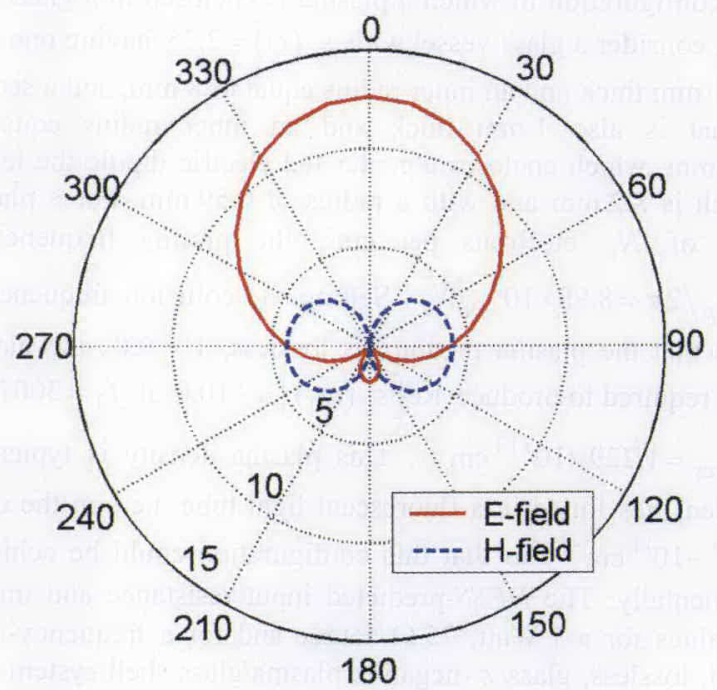

Figure 12c. The far-field $E$ - and $H$-field patterns for Design 1 .

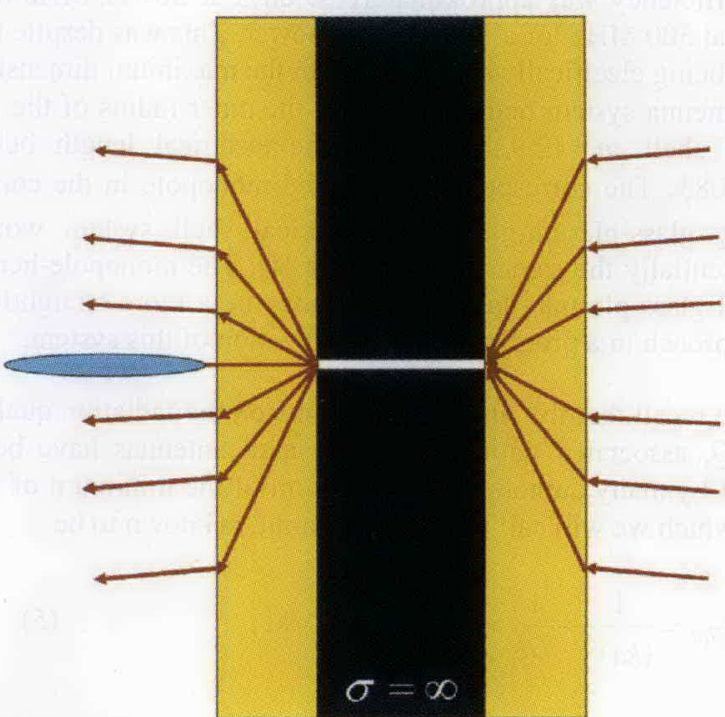

Figure 14. A heuristic ray-theory interpretation of the enhancement that can be obtained by using a metamaterial cover slab having an index of refraction less than that of free space on both sides of the hole. 
the real part of the magnetic field associated with the lossless version of this electrically small resonant system is illustrated in Figure $8 b$.

More-realistic electrically small center-fed electric dipole/ $\varepsilon$ negative spherical shell and coax-fed electric monopole/ $\varepsilon$-negative hemispherical shell antenna systems have also been modeled numerically using Ansoft's High Frequency Structure Simulator (HFSS) $[23,24]$. The input resistance and reactance of these realistic antenna/ $\varepsilon$-negative shell systems were obtained from these numerical models. It has been shown in $[23,24]$ that these systems could be designed to have geometrical resonances for which the relative gains were analogous to the radiated power ratios obtained with the analytical models. With further tuning, it has also been shown that an "antenna" resonance - where the system has a zero input reactance and an input resistance that is matched to a specified source resistance - can also be realized to yield a very good impedance match to the source. For example, consider a fiveregion configuration in which a plasma is enclosed in a glass vessel, i.e., consider a glass vessel with $\varepsilon_{r}(\omega)=2.25$ having one wall that is $1 \mathrm{~mm}$ thick and an inner radius equal to $8 \mathrm{~mm}$, and a second wall that is also $1 \mathrm{~mm}$ thick and an inner radius equal to $11.928 \mathrm{~mm}$, which encloses a center-fed electric dipole the length of which is $8.2 \mathrm{~mm}$ and with a radius of $0.59 \mathrm{~mm}$. For a plasma density of $N_{e}$ electrons per $\mathrm{cm}^{3}$, the plasma frequency is $f_{p}=\omega_{p} / 2 \pi=8.98 \times 10^{3} \sqrt{N_{e}}$. Setting the collision frequency to zero so that the plasma medium is lossless, $\Gamma=0.0$, the plasma density required to produce $\operatorname{Re}\left[\varepsilon_{r}\left(\omega_{0}\right)\right]=-10.0$ at $f_{0}=300 \mathrm{MHz}$ is $N_{e, r e s}=1.229 \times 10^{+10} \mathrm{~cm}^{-3}$. This plasma density is typical of those densities found in a fluorescent light tube, i.e., on the order of $10^{10}-10^{11} \mathrm{~cm}^{-3}$, so that this configuration could be achieved experimentally. The HFSS-predicted input resistance and impedance values for a 1 Watt, $75 \Omega$ source and for a frequency-independent, lossless, glass $/ \varepsilon$-negative plasma/glass shell system with $\varepsilon_{r}\left(\omega_{0}\right)=-10.0$ is shown in Figure 9. Values of $Z_{\text {input }}=72.159 \Omega$ at $307.22 \mathrm{MHz}$ and $Z_{\text {input }}=68.42-j 53.43 \Omega$ at $300.00 \mathrm{MHz}$ were found. Thus, for this hypothetically lossless system, the radiated power efficiency was approximately $99.86 \%$ at $307.22 \mathrm{MHz}$ and $87.62 \%$ at $300 \mathrm{MHz}$ for a $1 \mathrm{Watt}$ input power. This was despite the antenna being electrically small, i.e., with the maximum dimension of the antenna system being defined by the outer radius of the $\varepsilon$ negative shell, $a=12.928 \mathrm{~mm}$, and the electrical length being $k_{0} a=0.083$. The corresponding coax-fed monopole in the corresponding glass-plasma-glass hemispherical shell system would have essentially the same behavior $[23,24]$. The monopole-hemispherical glass-plasma-glass geometry may be a more straightforward approach to a proof-of-concept realization of this system.

We recall that the fundamental limits on the radiation quality factor, $Q$, associated with electrically small antennas have been explored by many authors. The exact form of the minimum of the $Q$, i.e., which we will call here the $\mathrm{Chu}$ limit, is known to be

$$
Q_{C h u}=\frac{1}{(k a)^{3}}+\frac{1}{k a} \approx \frac{1}{(k a)^{3}} \text { for } k a \ll 1,
$$

where $a$ is the radius of the radiansphere (minimum-radius sphere) surrounding the antenna system. On the other hand, if $f_{+, 3 d B}$ and

$f_{-, 3 d B}$ represent the frequencies above and below the resonance frequency where the radiated power falls to half its peak value, the fractional bandwidth, $F B W$, is related to the radiation quality fac- tor, $Q_{B W}$, by the relation $F B W=\Delta f_{3 d B} / f_{0 d B}=1 / Q_{B W} \approx(k a)^{3}$, where $\Delta f_{3 d B}=f_{+, 3 d B}-f_{-, 3 d B}$. Consequently, as the electrical size of the dipole antenna decreases, the minimum $Q$ value in free space increases dramatically, causing a corresponding decrease in the fractional bandwidth of the antenna. The quality factor and the fractional bandwidth are traditionally thought to approach their Chu limits only if the antenna efficiently utilizes the available volume within the radiansphere. When losses are present, the radiation efficiency, $\eta_{\text {rad }}$, and the quality factor for the lossy and lossless cases are related by $Q_{\text {Lossy }}=\eta_{\text {rad }} Q_{\text {Lossless }}$, i.e., the fractional bandwidth is increased in the presence of the loss at the cost of radiated power. Thus, the lossless cases are sufficient to bound the fractional bandwidth and quality factors of the system.

For the hypothetical nondispersive, lossless center-fed dipole/(glass- $\varepsilon$-negative plasma-glass) spherical-shell system, the input impedance of which is shown in Figure 9, the fractional halfpower VSWR bandwidth was $F B W_{V S W R}=13.16 \%$, and the corresponding quality factor was $Q_{V S W R}\left(\omega_{0}\right)=2 / F B W_{V S W R}\left(\omega_{0}\right)$ $=15.19$. The derivative values of the resistance and reactance curves in Figure 9 were also used to calculate the Yaghjian and Best formula for $Q$ [26]:

$$
Q_{Y B} \approx f_{0}\left|\partial_{f} Z_{\text {input }}\left(f_{0}\right)\right| / 2 R\left(f_{0}\right) \approx 15.53 \text {. }
$$

This value gave the so-called fractional conductance bandwidth, $F B W_{C D}=1 / Q_{Y B}=6.44 \%$, which was approximately half of the half-power VSWR fractional bandwidth value [26]. The analytical infinitesimal dipole-(glass-plasma-glass) spherical-shell system predicted the fractional bandwidth $F B W_{\text {Analytical }}=6.26 \%$ and the quality factor $Q_{\text {Analytical }}=15.964$. The corresponding Chu limit values with $k a=0.074936$ were $Q_{C h u}=2389.71$ and $F B W_{C h u}=0.042 \%$. When dispersion - which is always present in any realistic metamaterial - is taken into account, these metamaterial-based antenna $Q$ and bandwidth results change dramatically. Unfortunately, we were unable to investigate the corresponding dispersion effects with the HFSS models. The requisite very slight changes in frequency and the corresponding smaller changes in permittivity values are extremely difficult to resolve with the HFSS models, even on high-performance workstations. On the other hand, they are readily included in the analytical results. As this example shows and as was established in [23], the $Q$ results obtained with the analytical model of the ideal system were larger than those obtained from the numerical models of the more realistic system. We therefore feel that the use of the analytical models to study the effects of dispersion on the quality factors and bandwidths of these metamaterial-based antenna systems, checking with the numerical models whenever possible, is a reasonable approach.

The radiated power ratio values for the infinitesimal dipole(single-layer $\varepsilon$-negative) shell case associated with Figure 8, when the dispersive nature of the $\varepsilon$-negative medium was taken into account, are shown in Figure 10 as a function of the source frequency. For example, it was found that with a lossless Drude $\varepsilon$ negative medium model, $Q_{\text {Drude }}=3947.5 \approx 1.56 Q_{C h u}$ and $F B W_{\text {Drude }}=0.025 \% \approx 0.64 F B W_{C h u}$. However, for practical applications, one would ideally wish to achieve the quality factor and bandwidth associated with the non-dispersive, lossless $\varepsilon$-negative medium case: $Q_{\text {Const }}=14.43 \approx 0.0058 Q_{\text {Chu }}$ and $F B W_{\text {Const }}=6.93 \% \approx 173.03 F B W_{C h u}$. Can one achieve a band- 

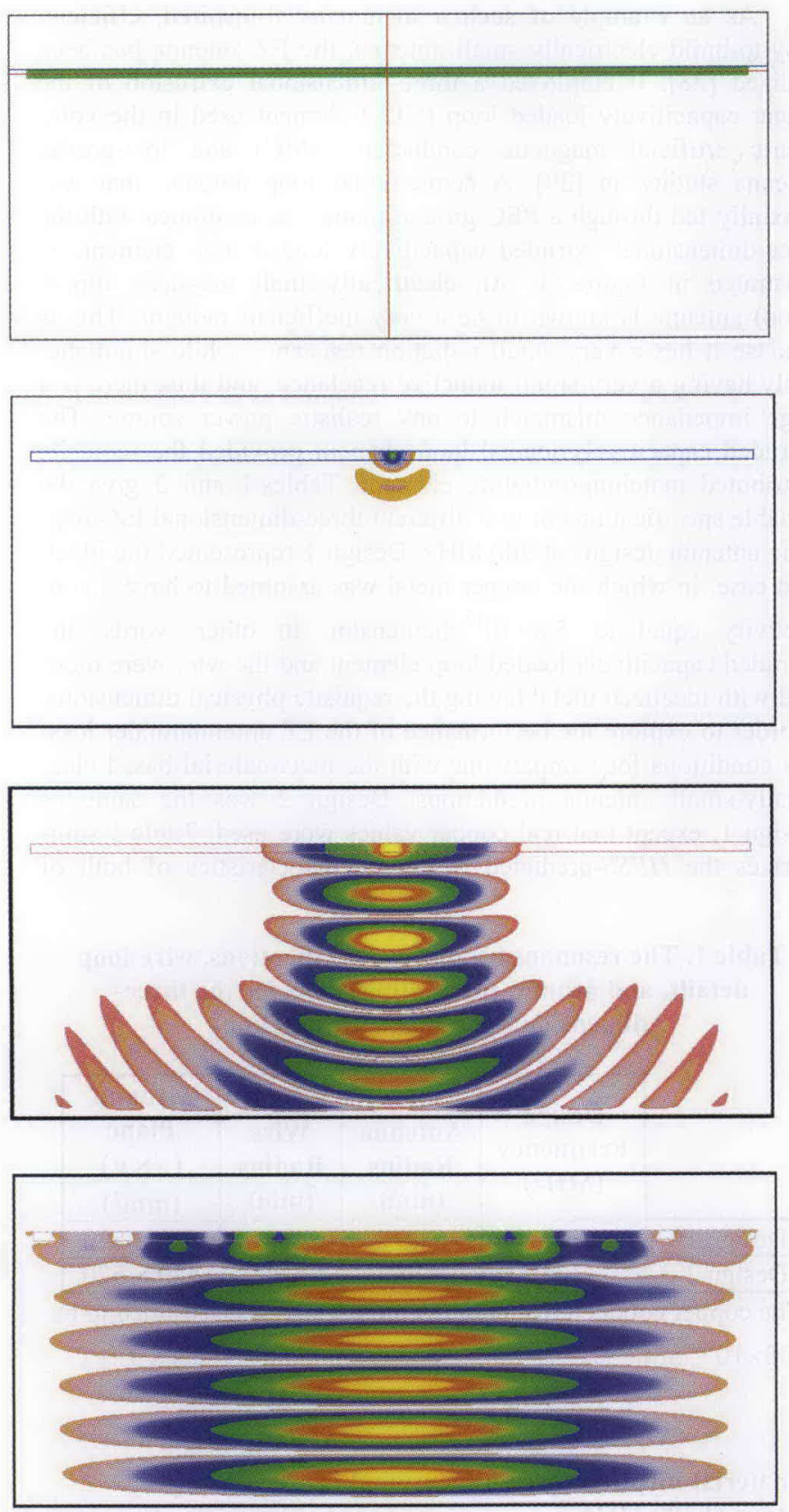

Figure 15. The electric-field intensity radiated by a line source centered in a $\lambda_{0} / 10$-thick zero-index slab that was terminated in a PMC sheet: a) $t=0$, b) $t=167 \Delta t$, c) $t=1000 \Delta t$, and d) $t=4833 \Delta t$ (from [37], @2005 The Institute of Electronics, Information, and Communications Engineers).

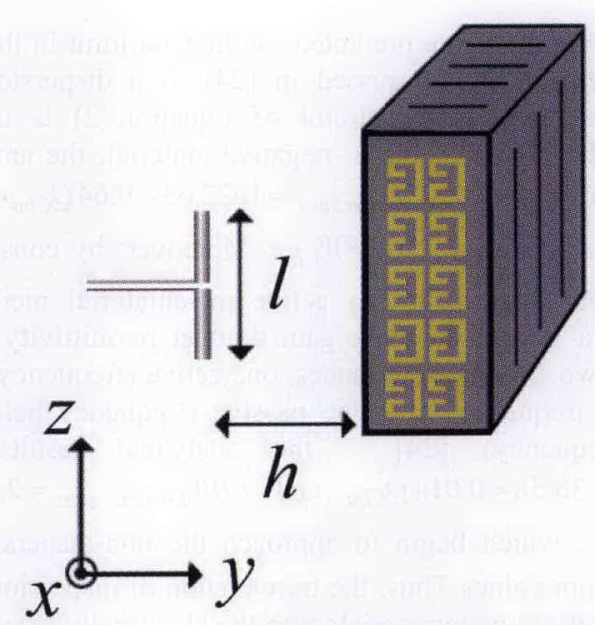

Figure 16a. The geometry of a capacitively loaded loop-based artificial magnetic conductor block, which will act as an inphase reflector for a low-profile dipole antenna near it.

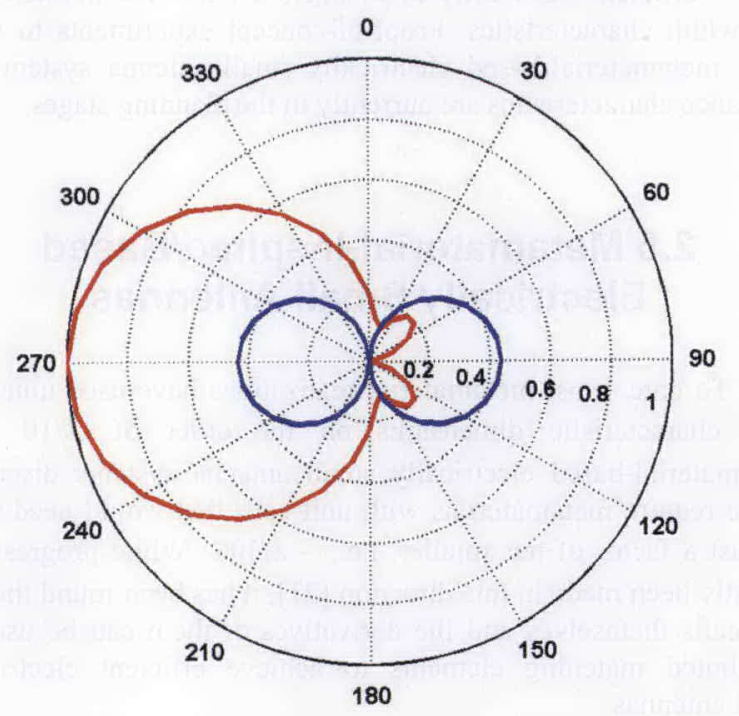

Figure 16b. The E-plane pattern for the geometry of Figure $16 \mathrm{a}$, compared to that of the dipole radiating in free space.

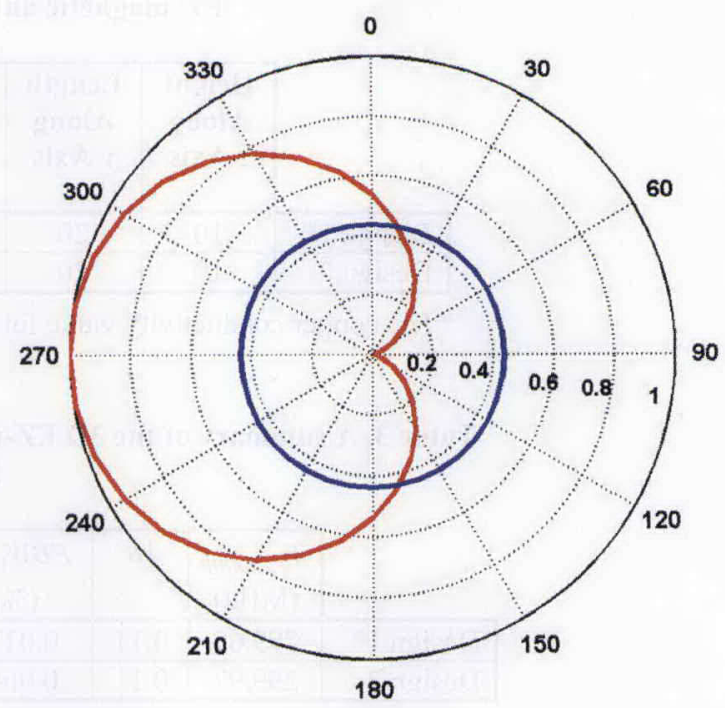

Figure 16c. The $H$-plane pattern for the geometry of Figure $16 \mathrm{a}$, compared to that of the dipole radiating in free space. 
width better than the value predicted by the Chu limit in the presence of dispersion? As discussed in [24], if a dispersion-limit model that satisfies the constraint of Equation (2) is used to describe the dispersion of the $\varepsilon$-negative material, the analytical model then gives $Q_{\text {Disp limit }}=1622.63 \approx 0.64 Q_{\text {Chu }}$ and $F B W_{\text {Displimit }}=0.062 \% \approx 1.56 F B W_{C h u}$. Moreover, by considering the $\varepsilon$-negative layer to be an active metamaterial medium described by a Lorentz-Lorentz gain doublet permittivity model created with two Lorentz resonances, one active (frequency above the operating frequency) and one passive (frequency below the operating frequency) [24] - the analytical results give $Q_{\text {Lorentz gain }}=35.58 \approx 0.014 Q_{C h u}$ and $F B W_{\text {Lorentz gain }}=2.81 \% \approx$ $71.33 \mathrm{FBW}_{\mathrm{Chu}}$, which begin to approach the non-dispersive $\varepsilon$ negative medium values Thus, the introduction of dispersion-engineered gain (active) metamaterials into the electrically small resonant dipole-multilayer $\varepsilon$-negative spherical-shell systems would allow one to realize bandwidths that would be quite interesting for a variety of applications. Consequently, by incorporating active metamaterials, there may in principle be a means of achieving not only an efficient electrically small antenna, but one with interesting bandwidth characteristics. Proof-of-concept experiments to study these metamaterial-based electrically small antenna system performance characteristics are currently in the planning stages.

\subsection{Metamaterial-Inspired/Based Electrically Small Antennas}

To date, most metamaterial realizations have used unit cells with characteristic dimensions on the order of $\lambda / 10$. The metamaterial-based electrically small antenna systems discussed above require metamaterials with unit cells that would need to be at least a factor of ten smaller, i.e., $\sim \lambda / 100$. While progress has recently been made in this direction [27], it has been found that the unit cells themselves and the derivatives of them can be used as distributed matching elements to achieve efficient electrically small antennas.
As an example of such a metamaterial-inspired, efficient, easy-to-build electrically small antenna, the EZ antenna has been realized [28]. It employed a three-dimensional extrusion of the planar capacitively loaded loop (CLL) element used in the volumetric artificial magnetic conductor (AMC) and low-profile antenna studies in [29]. A semicircular loop antenna that was coaxially-fed through a PEC ground plane was combined with the three-dimensional extruded capacitively loaded loop element, as illustrated in Figure 11. An electrically-small magnetic dipole (loop) antenna is known to be a very inefficient radiator. This is because it has a very small radiation resistance while simultaneously having a very small inductive reactance, and thus there is a large impedance mismatch to any realistic power source. The extruded capacitively loaded loop element provided the requisite distributed matching/radiating element. Tables 1 and 2 give the variable specifications of two different three-dimensional EZ-magnetic antenna designs at $300 \mathrm{MHz}$. Design 1 represented the idealized case, in which the copper metal was assumed to have a conductivity equal to $5.8 \times 10^{17} \mathrm{Siemens} / \mathrm{m}$. In other words, the extruded capacitively loaded loop element and the wire were modeled with idealized metal having the requisite physical dimensions, in order to explore the performance of the EZ antenna under lossless conditions for comparisons with the metamaterial-based electrically-small antenna predictions. Design 2 was the same as Design 1, except that real copper values were used. Table 3 summarizes the HFSS-predicted radiation characteristics of both of

Table 1. The resonant frequency specifications, wire-loop details, and ground-plane dimensions for the threedimensional EZ-magnetic antenna.

\begin{tabular}{|l|c|c|c|c|}
\cline { 2 - 5 } \multicolumn{1}{c|}{} & $\begin{array}{c}\text { Design } \\
\text { Frequency } \\
(\mathbf{M H z})\end{array}$ & $\begin{array}{c}\text { Loop } \\
\text { Antenna } \\
\text { Radius } \\
(\mathbf{m m})\end{array}$ & $\begin{array}{c}\text { Metal } \\
\text { Wire } \\
\text { Radius } \\
(\mathbf{m m})\end{array}$ & $\begin{array}{c}\text { Ground } \\
\text { Plane } \\
(x \times y) \\
\left(\mathbf{m m}^{\mathbf{2}}\right)\end{array}$ \\
\hline Design 1 & 300 & 1.9 & 0.3 & $520 \times 520$ \\
\hline Design 2 & 300 & 2.8 & 0.3 & $520 \times 520$ \\
\hline
\end{tabular}

*The copper conductivity value for this design was assumed to be $5.8 \times 10^{17} \mathrm{~S} / \mathrm{m}$.

Table 2. The dimensions (in $\mathrm{mm}$ ) of the metamaterial-inspired three-dimensional EZ-magnetic antenna structure at $300 \mathrm{MHz}$.

\begin{tabular}{|l|c|c|c|c|c|c|}
\cline { 2 - 6 } \multicolumn{1}{c|}{} & $\begin{array}{c}\text { Height } \\
\text { Along } \\
z \text { Axis }\end{array}$ & $\begin{array}{c}\text { Length } \\
\text { Along } \\
y \text { Axis }\end{array}$ & $\begin{array}{c}\text { Depth } \\
\text { Along } \\
x \text { Axis }\end{array}$ & $\begin{array}{c}\text { Spacing } \\
\text { Along } \\
y \text { Axis }\end{array}$ & $\begin{array}{c}\text { Stub } \\
\text { Length } \\
\text { Along } \\
z \text { Axis }\end{array}$ & $\begin{array}{c}\text { Copper } \\
\text { Metal } \\
\text { Thickness }\end{array}$ \\
\hline Design 1* & 10 & 20 & 20 & 0.03 & 5.741 & 0.254 \\
\hline Design 2 & 10 & 20 & 20 & 0.03 & 5.76 & 0.254 \\
\hline
\end{tabular}

*The copper conductivity value for this design was assumed to be $5.8 \times 10^{17} \mathrm{~S} / \mathrm{m}$.

Table 3. A summary of the 3D EZ-magnetic antenna's radiation characteristics at $300 \mathrm{MHz}^{* *}$.

\begin{tabular}{|l|c|c|c|c|c|c|c|c|}
\cline { 2 - 8 } \multicolumn{1}{c|}{} & $\begin{array}{c}F_{\text {resonant }} \\
(\mathrm{MHz})\end{array}$ & $k a$ & $\begin{array}{c}F B W_{V S W R} \\
(\%)\end{array}$ & $Q_{\text {ratio }}$ & $\begin{array}{c}A P \\
(\mathrm{~W})\end{array}$ & $\begin{array}{c}R E \\
(\%)\end{array}$ & $\begin{array}{c}O E \\
(\%)\end{array}$ & $D$ \\
\hline Design 1* & 299.69 & 0.11 & 0.0123 & 20.5 & 1 & 100 & 100 & 2.68 \\
\hline Design 2 & 299.97 & 0.11 & 0.0643 & 21.1 & 0.9969 & 18.73 & 18.67 & 2.68 \\
\hline
\end{tabular}

*The copper conductivity value for this design was assumed to be $5.8 \times 10^{17} \mathrm{~S} / \mathrm{m}$.

** $k a$ : Maximum electrical size of the antenna system; $A P$ : accepted power; $R E$ : radiation efficiency; $O E$ : overall efficiency; $D$ : directivity. 
these antenna systems. The half-power matched VSWR fractional bandwidth was used to compute the $Q$ value of each system at the resonance frequency, $f_{0}=\omega_{0} / 2 \pi$, i.e., $Q_{V S W R}\left(\omega_{0}\right)=2 / F B W_{V S W R}\left(\omega_{0}\right)$. The ratio, $Q_{\text {ratio }}$, of this $Q_{V S W R}$ value and the Chu-limit value, $Q_{C h u}$, obtained from Equation (5), was calculated to be

$$
Q_{\text {ratio }}\left(\omega_{0}\right)=2 /\left[F B W_{V S W R}\left(\omega_{0}\right) Q_{C h u}\left(\omega_{0}\right) \eta_{\text {rad }}\right] \text {, }
$$

where $\eta_{\text {rad }}$ is the radiation efficiency. The lossless-metal Design 1 produced a perfect $100 \%$ overall efficiency at $300 \mathrm{MHz}$ with $k a=0.11$. It represented a physical realization of the earlier theoretical predictions of a metamaterial-based electrically small antenna system with a high overall efficiency. It confirmed the ability of the extruded three-dimensional capacitively loaded loop element to act as a natural electrically-small distributed matching element. With realistic metal losses, the overall efficiency of the antenna system decreased to $18.67 \%$, a result of the large resonant fields in the presence of the lossy copper. It was found that near the electrically-small antenna limit, $k a=0.5$, the overall efficiencies of the lossy-copper versions of the three-dimensional EZ-magnetic antenna were around $96 \%$, and decreased as the value of $k a$ decreased because the resonant field strengths increased. The complex input impedance behavior, the predicted $S_{11}$ values for a $50 \Omega$ source, and the far-field radiation patterns obtained at $300 \mathrm{MHz}$ for Design 1 are shown in Figure 12. These results showed that the matching was very good; that it occurred as an anti-resonance, as expected from the magnetic nature of this system; and that the radiation patterns corresponded to a horizontal magnetic dipole over a PEC ground place.

It has been demonstrated [28] that this three-dimensional EZmagnetic antenna is linearly scalable to a wide range of frequencies. A two-dimensional interdigitated-capacitor version (i.e., where the three-dimensional gap capacitance is replaced by the interdigitated capacitor) of this antenna, which can also attain similar high overall efficiencies for a broad range of frequencies, has also been developed [30]. The dual two-dimensional and threedimensional EZ-electric antenna has also been considered, with similar outcomes. Designs at various frequencies have been considered. Experimental verification of the predictions for various two-dimensional EZ-magnetic and EZ-electric metamaterialinspired antennas have been obtained (see, e.g., [31]).

\section{Applications of ENZ and MNZ Materials}

While the combination of negative and positive materials may induce quasistatic interface resonances that lead to several exciting applications, as described in the previous sections, plasmonic materials with a low relative permittivity, low relative permeability, or low (or even a zero) index of refraction may show other interesting features. As for the dispersion requirements, it should be noted that materials with $\varepsilon$-near-zero or $\mu$-near-zero properties may have a larger bandwidth of operation when compared to negative-index materials, along with lower losses.

\subsection{Transparency Using Metamaterials}

Low-valued permittivities and/or permeabilities ensure a local negative electric and/or magnetic polarizability, with inter- esting potential applications. The polarization vectors $\mathbf{P}=\left(\varepsilon-\varepsilon_{0}\right) \mathbf{E}$ and $\mathbf{M}=\left(\mu-\mu_{0}\right) \mathbf{H}$ are, in fact, anti-parallel with the electric and magnetic fields if $\varepsilon<\varepsilon_{0}$ or $\mu<\mu_{0}$, respectively. This behavior may affect the scattering and radiating properties of objects made of such low-value materials. For example, if its polarizability is negative, the scattered field from an object the scattering cross section of which is dominated by the dipolar field will flip its phase. This implies that covering an object with a suitably designed cover may reduce the overall scattering cross section, making the covered object essentially transparent to an external observer, even in its near field [32]. This is in some manner the dual phenomenon of the resonant scattering described in the previous paragraph, even though this cancellation of the scattering relies on a different physical phenomenon. Not being a highly resonant phenomenon in this case, the sensitivity to losses and to the frequency variation is reduced and less pronounced than for the enhanced scattering of the complementary core-shell systems described above.

As an example, Figure 13 shows the scattered and total fields from a dielectric sphere of radius $\lambda_{0} / 4$ (on the left) and for the same sphere covered with an $\varepsilon$-near-zero cover (on the right), projected on a square screen of dimensions $6 \lambda_{0} \times 6 \lambda_{0}$. Similar results may be obtained for metallic objects and perfect conductors [32].

\subsection{Transmission Enhancement through Sub-Wavelength Apertures}

$\varepsilon$-near-zero and $\mu$-near-zero covers may be employed to drastically enhance the transmission through a sub-wavelength
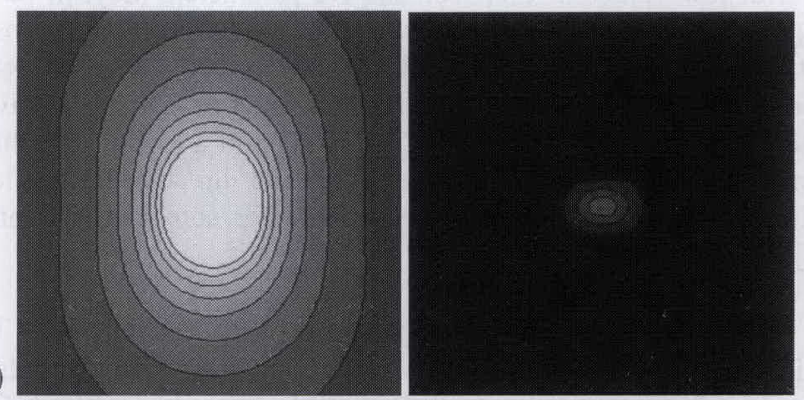

b)
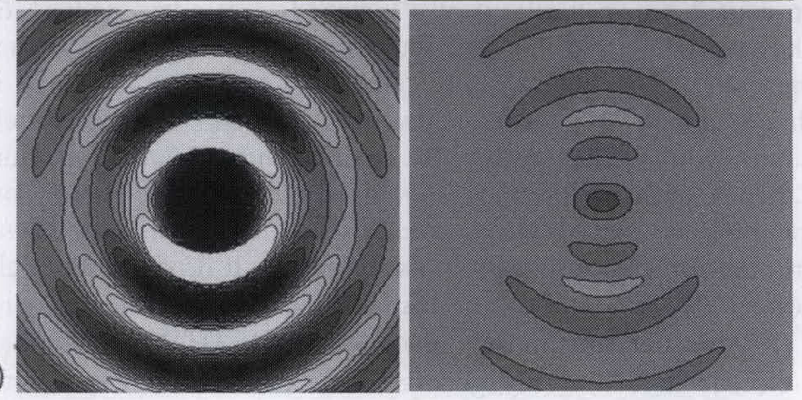

Figure 13. (a) Scattered and (b) total fields from a dielectric sphere of radius $\lambda_{0} / 4$ with permittivity $\varepsilon=10 \varepsilon_{0}$ (left column), and for the same sphere covered with an $\varepsilon$-near-zero material (right column), designed following the theory described in [32], projected on a square screen of dimensions $6 \lambda_{0} \times 6 \lambda_{0}$ placed $0.5 \lambda_{0}$. The field levels are at the same scale in the left and right columns. 
aperture in a flat opaque screen, overcoming the diffraction limit. An isolated sub-wavelength aperture generally does not provide good power transmission, which, in the quasi-static limit, is proportional to the fourth power of its electrical size. Covering the hole and the screen with properly designed materials at the entrance and exit side of the aperture, the transmission may be enhanced by several orders of magnitude. The enhancement of the transmitted power relies on the fact that the grounded $\varepsilon$-near-zero and $\mu$-near-zero layers support highly directive leaky waves. As reported in [33], these leaky waves can collect the impinging power at the entrance side of the screen, then tunnel it through the tiny aperture, further help this tunneling at the exit side, and finally reshape the beam to enhance its directivity towards a preferred direction. The combination of these effects may dramatically enhance the total transmitted power.

Figure 14 shows a heuristic picture of this enhancement phenomenon, explained in terms of ray theory. Due to the low refraction index inside the materials, rays are bent towards the normal at the interface between the low-positive material and free space. This enhances the power reception on the entrance side and the directivity at broadside on the exit side.

\subsection{Applications to Antenna Setups}

The focusing properties of materials with a low index of refraction may be applied to antenna problems to improve their performance. The concepts exploited in the previous paragraph may be directly applied to build leaky-wave antennas, as has been proposed in [34]. When combined with the phase-compensation effects exploited in the previous paragraph, this may provide a new generation of leaky-wave antennas, with low profile and high directivity. It has been shown that single-negative grounded bi-layers with low values of their constitutive parameters may, in fact, support highly directive leaky waves in a sub-wavelength cross section. A similar effect has also been studied in cylindrical geometries, and a coaxial sub-wavelength shell of low- $\varepsilon$-negative material has been theoretically shown to support a leaky wave that is omnidirectional in the azimuthal plane and directive in the elevation angle, and which may be suitable for some applications [35].

The use of $\varepsilon$-near-zero and $\mu$-near-zero metamaterials to achieve highly directional properties of the radiated fields has also been considered in the limit of a matched zero-index media [36]. By having the permittivity and permeability simultaneously both at or near zero, the medium is matched to free space. A source in such a medium will generate essentially a spatially-static field structure, which has a uniform phase value throughout the medium and, hence, at the output face. Moreover, there is no reflection loss at the interface, since the source and output media are matched. An aperture with a uniform phase distribution will produce the highest-directivity output beam. This behavior is illustrated in Figure 15, with snapshots in time predicted by an FDTD simulation of the electric-field intensity generated by a line source in a zeroindex slab that is backed with a perfect magnetic conductor (PMC) surface (the PMC surface does not short out the uniform electric field in the slab) [37]. The fields propagated away from the source, creating a spatially uniform field in the zero-index medium once steady state was reached. At that time, the output beam was generated from a uniform field distribution across the entire output face of the finite slab. This behavior has been demonstrated with electromagnetic bandgap (EBG) structures [38].

\subsection{Large ENG or MNG Material Responses}

Another antenna application for metamaterials that has stimulated much interest is the concept of an artificial magnetic conductor, i.e., an in-phase reflector. This can be achieved with a $\mu$ negative material with a large permeability or with an $\varepsilon$-near-zero material. A wide variety of artificial-magnetic-conductor (AMC) realizations has been considered, and these include mushroom surfaces $[39,40]$, frequency-selective surfaces (FSSs) and EBG surfaces [40, 41], and space-filling curves [42]. All of these classes of artificial magnetic conductors include a PEC ground plane, and thus at least guarantee high reflectivity. In contrast, in [29] an artificial magnetic conductor was realized with only the capacitivelyloaded-loop (CLL) -based $\mu$-negative material shown in Figure 16 a, i.e., there was no ground plane. This aspect demonstrated that the response of the $\mu$-negative material can be made large enough by itself to realize the requisite in-phase reflectivity. Moreover, the associated surface modes are dramatically different from those associated with the ground-plane varietals. These artificial magnetic conductors are highly desired for achieving low-profile antennas, i.e., an antenna as shown in Figure 16a can be placed in very close proximity to the artificial magnetic conductor without it being shorted out, as it would be in the presence of a PEC. The predicted radiation patterns for a dipole antenna near the capacitively-loaded-loop-based artificial magnetic conductor block are compared to those of a dipole radiating in free space in Figures $16 \mathrm{~b}$ and $16 \mathrm{c}$. Current investigations into these various artificial-magnetic-conductor surfaces include decreasing their thicknesses; achieving active substrates to realize multi-band and tunable artificial magnetic conductors; achieving very high front-toback ratios; and various aspects of array applications, such as beam steering and the mitigation of scan blindness.

\section{Conclusions}

Materials with anomalous values of their constitutive parameters have been shown to possess interesting potential in the design and engineering of next-generation structures for guiding, scattering, and radiating applications. Resonances arising in electrically small regions of space, where negative materials are paired with common dielectrics, present great potential for overcoming the limits generally associated with several electromagnetic problems, by providing a means to engineer the overall responses of the systems. Low-refraction materials, despite their non-resonant character, may also have a strong impact in some applications, since they combine anomalous wave interaction with relatively larger bandwidth and lower losses.

The metamaterials research area has evolved into prominence only very recently. Nonetheless, it is already having a large impact on our electromagnetics community. Metamaterials have revitalized our interests in complex media, and in their analysis and numerical modeling. There have been large strides in our understanding of the anomalous behaviors of these materials, and in our potential utilization of their exotic properties in many electromagnetic applications, from the microwave to the optical regime. We have briefly reviewed here some of what has already been learned about several aspects of these topics; we look forward to further progress in the future. 


\section{Acknowledgments}

The collections of works summarized here have been supported by several grants from DARPA, including contract numbers HR0011-05-C-0068, HR0011-04-P-0042, and N000140510862.

\section{References}

1. C. Bohren and D. Huffmann, Absorption and Scattering of Light by Small Particles, New York, Wiley, 1983.

2. N. Engheta and R. W. Ziolkowski (eds.), "Special Issue on Metamaterials," IEEE Transactions on Antennas and Propagation, AP-51, October 2003, pp. 2546-2750.

3. L. Landau, and E. M. Lifschitz, Electrodynamics of Continuous Media, Oxford, UK, Pergamon Press, 1984.

4. R. W. Ziolkowski and E. Heyman, "Wave Propagation in Media Having Negative Permittivity and Permeability," Phys. Rev. E, 64, 056625, October 2001, pp. 1-15.

5. J. B. Pendry, A. J. Holden, D. J. Robbins, and W. J. Stewart, "Magnetism from Conductors and Enhanced Nonlinear Phenomena," IEEE Transactions on Microwave Theory and Techniques, MTT-47, 11, November 1999, pp. 2075-2081.

6. R. W. Ziolkowski, "Propagation in and Scattering from a Matched Metamaterial Having a Zero Index of Refraction," Phys. Rev. E, 70, 046608, October, 2004.

7. R. W. Ziolkowski and A. Kipple, "Causality and Double-Negative Metamaterials," Phys. Rev. E, 68, 026615, August 29, 2003.

8. A. Alù, N. Engheta, R. W. Ziolkowski, and A. Erentok, "Fundamentals of Waveguide and Antenna Applications Involving DNG and SNG Metamaterials," in N. Engheta and R. Ziolkowski (eds.), Electromagnetic Metamaterials: Physics and Engineering Explorations, New York, IEEE/John Wiley, 2006.

9. N. Engheta, "An Idea for Thin, Subwavelength Cavity Resonators Using Metamaterials with Negative Permittivity and Permeability," IEEE Antennas and Wireless Propagation Letters, 1, 2002, pp. 10-13.

10. A. Alù and N. Engheta, "Guided Modes in a Waveguide Filled with a Pair of Single-Negative (SNG), Double-Negative (DNG), and/or Double-Positive (DPS) Layers," IEEE Transactions on Microwave Theory and Techniques, MTT-52, 2004, pp. 199-210.

11. N. Engheta and R. W. Ziolkowski, "A Positive Future for Double-Negative Metamaterials," IEEE Transactions on Microwave Theory and Techniques, MTT-53, April 2005, pp. 1535-1556.

12. R. W. Ziolkowski, "Ultra-Thin Metamaterial-Based Laser Cavities,” J. Opt. Soc. Am. B, 23, 3, March 2006, pp. 451-460.

13. A. Alù and N. Engheta, "An Overview of Salient Properties of Planar Guided-Wave Structures with Double-Negative (DNG) and Single-Negative (SNG) Layers," in G. V. Eleftheriades and K. G. Balmain (eds.), Negative Refraction Metamaterials: Fundamental Properties and Applications, New York, IEEE Press/John Wiley, 2005, pp. 339-380.
14. N. Engheta and A. Alù, "May Cavities and Waveguides be Ultra-Thin and Still Support Resonant Modes when they Contain Double-Negative (DNG) or Single-Negative (SNG) Media?" Proceedings of the Progress in Electromagnetics Research Symposium (PIERS'03), Waikiki, Hawaii, USA, October 13-16, 2003, p. 381.

15. A. Alù and N. Engheta "Anomalies in the Surface Wave Propagation Along Double-Negative and Single-Negative Cylindrical Shells," Proceedings of the Progress in Electromagnetics Research Symposium (PIERS'04), Pisa, Italy, CD Digest, March 28-31, 2004.

16. A. Alù and N. Engheta, "Pairing an Epsilon-Negative Slab with a Mu-Negative Slab: Anomalous Tunneling and Transparency," IEEE Transactions on Antennas and Propagation, AP-51, 10, October 2003, pp. 2558-2570.

17. A. Alù, N. Engheta, and R. W. Ziolkowski, "FDTD Simulations of Tunneling and 'Growing Exponential' in a Pair of \&-Negative and $\mu$-Negative Slabs," submitted to Phys. Rev. E.

18. A. Alù and N. Engheta, "Polarizabilities and Effective Parameters for Collections of Spherical Nano-Particles Formed by Pairs of Concentric Double-Negative (DNG), Single-Negative (SNG) and/or Double-Positive (DPS) Metamaterial Layers," Journal of Applied Physics, 97, 094310 (12 pages), May 1, 2005.

19. N. Engheta and A. Alù, "Can Negative-Parameter Metamaterials Provide High Directivity for Small Apertures and Antennas?" Digest of the USNC/URSI National Radio Science Meeting, Washington, DC, USA, July 3-8, 2005, p. 99.

20. J. A. Gordon and R. W. Ziolkowski, "The Design and Simulated Performance of a Coated Nano-Particle Laser," to appear in Opt. Exp., 2007.

21. R. W. Ziolkowski and A. Kipple, "Application of Double Negative Metamaterials to Increase the Power Radiated by Electrically Small Antennas," IEEE Transactions on Antennas and Propagation, AP-51, October 2003, pp. 2626-2640.

22. R. W. Ziolkowski and A. D. Kipple, "Reciprocity Between the Effects of Resonant Scattering and Enhanced Radiated Power by Electrically Small Antennas in the Presence of Nested Metamaterial Shells," Phys. Rev. E., 72, 036602, September 2005.

23. R. W. Ziolkowski and A. Erentok, "Metamaterial-Based Efficient Electrically Small Antennas," IEEE Transactions on Antennas and Propagation, AP-54, July 2006, pp. 2113-2130.

24. R. W. Ziolkowski and A. Erentok, "At and Below the Chu Limit: Passive and Active Broad Bandwidth Metamaterial-Based Electrically Small Antennas," to appear in the IET Proceedings, 2007.

25. A. Erentok and R. W. Ziolkowski, "A Hybrid Optimization Method to Analyze Metamaterial-Based Electrically Small Antennas," to appear in IEEE Transactions on Antennas and Propagation, 2007.

26. A. D. Yaghjian and S. R. Best, "Impedance, Bandwidth, and Q of Antennas," IEEE Transactions on Antennas and Propagation, AP-53, 4, April 2005, pp. 1298-1324.

27. P. D. Imhof, R. W. Ziolkowski, and J. R. Mosig, "Highly Subwavelength Unit Cells to Achieve Epsilon Negative (ENG) Meta- 
material Properties," Proceedings of the 2006 IEEE AP-S International Symposium and USNC/URSI National Radio Science Meeting, Albuquerque, NM, July 9-14, 2006.

28. A. Erentok and R. W. Ziolkowski, "An Efficient MetamaterialInspired Electrically-Small Antenna," to appear in Microwave Opt. Tech. Lett., 2007.

29. A. Erentok, P. Luljak, and R. W. Ziolkowski, "Antenna Performance Near a Volumetric Metamaterial Realization of an Artificial Magnetic Conductor," IEEE Transactions on Antennas and Propagation, AP-53, January 2005, pp. 160-172.

30. A. Erentok and R. W. Ziolkowski, "Two-Dimensional Efficient Metamaterial-Inspired Electrically-Small Antenna," to appear in Microwave Opt. Tech. Lett., 2007.

31. A. Erentok and R. W. Ziolkowski, "Efficient MetamaterialInspired Electrically-Small Electric-Based Antennas: Two- and Three-Dimensional Realizations," submitted to the 2007 IEEE International Symposium on Antennas and Propagation, January 2007.

32. A. Alù, and N. Engheta, "Achieving Transparency with Plasmonic and Metamaterial Coatings," Physical Review E, 72, 016623 (9 pages), July 26, 2005.

33. A. Alù, F. Bilotti, N. Engheta, and L. Vegni, "How Metamaterials May Significantly Affect the Wave Transmission Through Sub-Wavelength Hole in a Flat Perfectly Conducting Screen," Proceedings of IEE Seminar on Metamaterials for Microwave and (Sub) millimetre Wave Applications: Photonic Bandgap and Double Negative Designs, Components and Experiments, London, UK, November 24, 2003, pp. 11/1-11/6.

34. A. Alù, F. Bilotti, N. Engheta, and L. Vegni, "Compact LeakyWave Components Using Metamaterials," Proceedings of the IEEE MTT-S 2005 International Microwave Symposium (IMS'05), Long Beach, California, USA, CD Digest, June 12-17, 2005.
35. A. Alù, F. Bilotti, N. Engheta, and L. Vegni, "Anomalous Radiation Properties of Cylindrical Metamaterial Leaky-Wave Antennas," Proceedings of the International Conference on Electromagnetics in Advanced Applications (ICEAA'05), Turin, Italy, September 12-16, 2005, pp. 575-578.

36. R. W. Ziolkowski, "Propagation in and Scattering from a Matched Metamaterial Having a Zero Index of Refraction," Phys. Rev. E, 70, 046608, October, 2004.

37. R. W. Ziolkowski, "Antennas and Propagation in the Presence of Metamaterials and Other Complex Media: Computational Electromagnetic Advances and Challenges," IEICE Trans. Electron., E88-B, 6, June 2005, pp. 2230-2238.

38. B. Gralak, S. Enoch, and G. Tayeb, "Superprism Effects and EBG Antenna Applications," in N. Engheta and R. Ziolkowski (eds.), Electromagnetic Metamaterials: Physics and Engineering Explorations, New York, IEEE/John Wiley, 2006.

39. D. Sievenpiper, "A Review of the Theory, Fabrication, and Applications of High-Impedance Ground Planes," in N. Engheta and R. Ziolkowski (eds.), Electromagnetic Metamaterials: Physics and Engineering Explorations, New York, IEEE/John Wiley, 2006.

40. Y. Rahmat-Samii and F. Yang, "Development of Complex Artificial Ground Planes for Antenna Engineering," in N. Engheta and R. Ziolkowski (eds.), Electromagnetic Metamaterials: Physics and Engineering Explorations, New York, IEEE/John Wiley, 2006.

41. S. Maci, and A. Cucini, "FSS-based EBG Surfaces," in N. Engheta and R. Ziolkowski (eds.), Electromagnetic Metamaterials: Physics and Engineering Explorations, New York, IEEE/John Wiley, 2006.

42. J. McVay, N. Engheta, A. Hoorfar, "A Review of the Theory, Fabrication, and Applications of High-Impedance Ground Planes," in N. Engheta and R. Ziolkowski (eds.), Electromagnetic Metamaterials: Physics and Engineering Explorations, New York, IEEE/John Wiley, 2006.

\section{Deadline Extended}

The paper-submission deadline for the Second European Conference on Antennas and Propagation (EuCAP), November 1116, 2007, Edinburgh, UK has been extended until April 16, 2007. 